\title{
TP53-induced glycolysis and apoptosis regulator is indispensable for mitochondria quality control and degradation following damage
}

\author{
JING FENG $^{1 *}$, LI LUO ${ }^{2 *}$, YONG LIU ${ }^{1}$, SHAOZHI FU ${ }^{1}$, JIE CHEN $^{3}$, XIAOXIA DUAN ${ }^{4}$, \\ LI XIANG $^{1}$, YANLING ZHANG ${ }^{1}$, JINBO WU ${ }^{1}$, JUAN FAN ${ }^{1}$, QINGLIAN WEN ${ }^{1}$, \\ YE ZHANG $^{5}$, JINGPIN YANG ${ }^{1}$, JINXIA PENG ${ }^{1}$, MING ZHAO $^{1}$ and LINGLIN YANG ${ }^{1}$
}

Departments of ${ }^{1}$ Oncology, ${ }^{2}$ Laboratory Medicine, ${ }^{3}$ Rheumatology and Immunology, ${ }^{4}$ Anesthesiology and
${ }^{5}$ Neurosurgery, The Affiliated Hospital of Southwest Medical University, Luzhou, Sichuan 646000 , P.R. China

Received September 13, 2016; Accepted September 22, 2017

DOI: $10.3892 / 01.2017 .7303$

\begin{abstract}
Mitochondria have been described as 'the powerhouse of the cell' as the organelle generates the majority of adenosine triphosphate (ATP) in cells to support life. Mitochondria can be damaged due to stress, for example by reactive oxygen species (ROS). TP53-induced glycolysis and apoptosis regulator (TIGAR) serves a role in suppressing ROS damage and may protect mitochondria integrity. In the present study, the localization of TIGAR on mitochondria in 5-8F cells was demonstrated. Furthermore, it was indicated that the knockdown of TIGAR using lentivirus-short hairpin RNA induces the loss of mitochondrial membrane potential and cytochrome c leakage. However, these damaged mitochondria were not degraded in cells, but exhibited an abnormal appearance as indicated by mitochondrial swelling, crista collapse and vacuolization, with physiological dysfunction marked by reduced ATP production. Therefore, TIGAR maybe an indispensable protein for mitochondrial protection and degradation following cellular damage.
\end{abstract}

\section{Introduction}

Mitochondria serve a crucial role in the determination of cellular life and death. Mitochondria are essential for energy production, as most of the cellular adenosine triphosphate

Correspondence to: Dr Ming Zhao or Professor Linglin Yang, Department of Oncology, The Affiliated Hospital of Southwest Medical University, 25 Taiping Street, Luzhou, Sichuan 646000, P.R. China

E-mail: zhaoming24@126.com

E-mail: yangllluyi@126.com

*Contributed equally

Key words: TP53 induced glycolysis regulatory phosphatase, mitochondrial membrane potential, mitochondrial degradation, mitochondrial integrity
(ATP) is produced by the oxidative phosphorylation pathway in the mitochondrial matrix (1). Mitochondria are fundamental players in numerous cellular activities, which include cellular signaling, generation and regulation of reactive oxygen species (ROS) levels, buffering cytosolic calcium levels and regulation of apoptosis via the mitochondrial permeability transition pore (mtPTP) (2,3). Mitochondrial dysfunction has been implicated in metabolic diseases, including cancer and aging (4-6). Therefore, mitochondrial quality control and function integrity are essential for cellular homeostasis.

Accumulating evidence has revealed that damaged mitochondria can be selectively removed by mitophagy, which is a specialized form of autophagy. During mitophagy, specific regulators, including phosphatase and tensin homolog-induced putative kinase 1 (PINK1) and Parkin, ensure selective sequestration of individual mitochondria as cargo (7). Mitochondrial depolarization, caused by the loss of mitochondrial membrane potential, may trigger mitophagy. During mitophagy, PINK1 and the E3 ubiquitin ligase Parkin accumulate on damaged mitochondria and thereby facilitate their segregation from the mitochondrial network. PINK1 and Parkin then target and facilitate ubiquitination of mitochondrial proteins that interact with various components of the core autophagy machinery (8-10). A double-membrane structure, known as the autophagosome, is then formed and fuses with the lysosome to form an autolysosome, in which the clearance of mitochondria will occur (7).

TIGAR has been determined as a fructose-2,6-bisphosphatase that lowers glycolytic flux and promotes antioxidant function. The reduction of glycolytic flux promotes the pentose phosphate pathway (PPP), which generates NADPH and increases the level of reduced glutathione, which aids to limit ROS production (11). The downregulation of TIGAR is linked to decreased levels of NADPH, lower levels of reduced glutathione, and consequently an increase in endogenous ROS (12). ROS, including superoxide $\left(\mathrm{O}_{2}^{-}\right)$, hydrogen peroxide $\left(\mathrm{H}_{2} \mathrm{O}_{2}\right)$ and hydroxyl radical $\left(\mathrm{OH}^{-}\right)(13)$, can attack mitochondria and induce mitochondrial DNA mutation and damage to cellular DNA and proteins (6).

In the present study, it was hypothesized that the expression of TIGAR in 5-8F nasopharyngeal carcinoma cells may 
affect mitochondrial integrity and degradation. Therefore, the role of TIGAR on mitophagy was investigated by transfecting 5-8F cells with lentivirus-mediated small hairpin RNA (lenti-shRNA) that targets the TIGAR gene to knockdown the expression of TIGAR. In the present study, it was determined that the knockdown of TIGAR initiates mitophagy that is associated with loss of mitochondrial membrane potential and cytochrome c leakage. In addition, in the present study, it was revealed that the knockdown of TIGAR prevents mitochondrial degradation, as indicated by the increase in mitochondrial mass due to swollen mitochondria.

\section{Materials and methods}

Cell lines and reagents. The 5-8F human nasopharyngeal carcinoma cell line was obtained from the American Type Culture Collection (Manassas, VA, USA). The cell routinely maintained in RPMI-1,640 medium (Gibco; Thermo Fisher Scientific, Inc., Waltham, MA, USA) supplemented with $10 \%$ fetal bovine serum (GE Healthcare Life Sciences, Little Chalfont, UK), $100 \mathrm{U} / \mathrm{ml}$ penicillin and $100 \mu \mathrm{g} / \mathrm{ml}$ streptomycin (Beyotime Institute of Biotechnology, Haimen, China) at $37^{\circ} \mathrm{C}$ in $5 \% \mathrm{CO}_{2}$. The cells were treated with Cyclosporin A (CsA; Sigma-Aldrich; Merck KGaA, Darmstadt, Germany) or protonophore carbonyl cyanide $\mathrm{m}$-chlorophenyl hydrazine (CCCP; Sigma-Aldrich; Merck KGaA) for $2 \mathrm{~h}$ in $37^{\circ} \mathrm{C}$, prior to detection at a final concentration of 5 and $10 \mu \mathrm{M}$, respectively.

Lenti-shRNA against TIGAR. The lenti-shRNA vector system against TIGAR was constructed, packed, and purified by GeneChem, Inc. (Shanghai, China) according to the protocol of the manufacturer. The shRNA oligonucleotides were designed as sh-TIGAR (5'-GCCAGCTTTACTGGAGAA CTT-3'). A scramble sequence (sh-scramble) was synthesized and served as a transfection control (5'-TTACCGAGACCG TACGTAT-3'). The virus titer was measured by GeneChem, Inc. To experimental measurement multiplicities of infection (MOI), four groups were created: MOI 1, 10, 50 and 100, respectively. Cells were transfected in complete medium (Gibco; Thermo Fisher Scientific, Inc.) with $5 \mu \mathrm{g} / \mathrm{ml}$ polybrene (cat no. REVG0001; GeneChem, Inc.) and 0.5, 5, 25 and $50 \mu \mathrm{l}$-viral particles respectively for $72 \mathrm{~h}$ at $37^{\circ} \mathrm{C}$. Then, the medium was replaced and the transfected cells were cultured with puromycin (Sigma-Aldrich; Merck KGaA) for 3-5 days in $37^{\circ} \mathrm{C}$. Subsequently, the cells with higher survival rate and better cell state were selected using light microscopy (Leica DMLS; Leica Microsystems) at x200 magnification, which corresponded to a suitable MOI value. The experiment revealed the most suitable MOI was 10 . Following transduction of 5-8F cells, colonies expressing a stable shRNA were selected using puromycin (Sigma-Aldrich; Merck KGaA), according to the manufacturer's protocol.

Western blotting. Total proteins from cells were extracted by radioimmunoprecipitation buffer with protease inhibitor (Beyotime Institute of Biotechnology, Haimen, China), then proteins (20-40 $\mu \mathrm{g}$ per lane) were separated on an SDS-PAGE gel (separating gel, 10 or $12 \%$; stacking gel, $5 \%$ ), then proteins were transferred to a polyvinylidene difluoride membrane (GE Healthcare, Chicago, IL, USA). Followed by blocking with 5\% milk at room temperature for $1 \mathrm{~h}$ and then incubation with the following antibodies: anti-TIGAR (1:400; cat no. sc-166290; Santa Cruz Biotechnology, Inc., Dallas, TX, USA); anti-GAPDH (1:1,000; cat no. 2118; Cell Signaling Technology, Inc., Danvers, MA, USA); anti-GRP75 (1:1,000; cat no. 14887-1-AP; ProteinTech Group, Inc., Chicago, IL, USA); anti-Cytochrome c (1:200; cat no. AC909; Beyotime Institute of Biotechnology, Haimen, China) overnight at $4^{\circ} \mathrm{C}$. The next day, membranes were washed with TBST and incubated with goat anti-rabbit, horseradish peroxidase-conjugated secondary antibodies (1:3,000; cat no. SA00001-2; ProteinTech Group, Inc., Chicago, IL, USA) for $1-2 \mathrm{~h}$ at room temperature. Then, the electrochemiluminescence peroxidase substrate (catalog no., WBLUR0100; EMD Millipore, Billerica, Ma, USA) was used (GE Healthcare Life Sciences) for the detection of immunoblotting on the ChemiDoc $^{\mathrm{TM}}$ MP Imaging System (Bio-Rad Laboratories, Inc.). The densitometry was analysed by the Image $\mathrm{Lab}^{\mathrm{TM}}$ software (Version 5.2.1, Bio-Rad Laboratories, Inc.).

Immunofluorescence analysis. The cells were seeded on a coverslip in a 24 -well plate at a density of $2 \times 10^{4}$ cells/well. In brief, the cells were fixed with $4 \%$ paraformaldehyde for $10 \mathrm{~min}$ at room temperature, then blocked with $3 \%$ goat serum (Beijing Solarbio Science \& Technology Co., Ltd., Beijing, China) for $1 \mathrm{~h}$ at room temperature and incubated with a primary anti-TIGAR mouse antibody (1:200; cat no. sc-166290; Santa Cruz Biotechnology, Inc.) and anti- mitochondrial heat shock protein 70 (mtHSP70) rabbit antibody (1:400; cat no; 14887-1-AP; ProteinTech Group, Inc.) overnight at $4^{\circ} \mathrm{C}$. The cells were washed twice in phosphate-buffered saline (PBS) and incubated with Cy3-labeled goat anti-mouse $\operatorname{IgG}(\mathrm{H}+\mathrm{L})$ and Cy2-labeled goat anti-rabbit IgG $(\mathrm{H}+\mathrm{L})$ antibodies (1:400; cat nos. A0521 and A0423; Beyotime Institute of Biotechnology) for $1 \mathrm{~h}$ at room temperature. Then cells were washed twice in PBS and observed under a fluorescence microscope.

Flowcytometry. For flow cytometry experiments, the cells were seeded in a 6-well plate at a density of $15 \times 10^{4}$ cells/well. A total of $24 \mathrm{~h}$ after the start of inoculation, the medium was replaced with Hank's balanced salt solution-containing Mito-Tracker Green (200 nM; Beyotime Institute of Biotechnology, Haimen, China). Following incubation for $30 \mathrm{~min}$ at $37^{\circ} \mathrm{C}$, the cells were washed twice with PBS and resuspended in $500 \mu \mathrm{l}$ PBS prior to analysis by flow cytometry (FACSVerse; FACSuite ${ }^{\mathrm{TM}}$ v. 1.0.3.2942 software; BD Biosciences, Franklin Lakes, NJ, USA).

Mitochondrial membrane potential assay. For mitochondrial membrane potential assays, the cells were seeded in a 6-well plate at a density of $15 \times 10^{4}$ cells/well. A total of $24 \mathrm{~h}$ after the start of the inoculation, the cells were stained for $30 \mathrm{~min}$ with $15 \mu \mathrm{mol} / 15,5$ ', 6,6'-tetrachloro-1,1', 3,3'-tetraethyl-imidacarbocyanineiodide (JC-1; Beyotime Institute of Biotechnology) in cell culture media at $37^{\circ} \mathrm{C}$ followed by two washes with staining buffer (Beyotime Institute of Biotechnology) prior to analysis using a fluorescence microscope (magnification, $\mathrm{x} 400$ ). The ratio between green and red fluorescence signals served as a parameter for the mitochondrial membrane potential $(\Delta \Psi m)$ and is independent of the mitochondrial mass. 
A
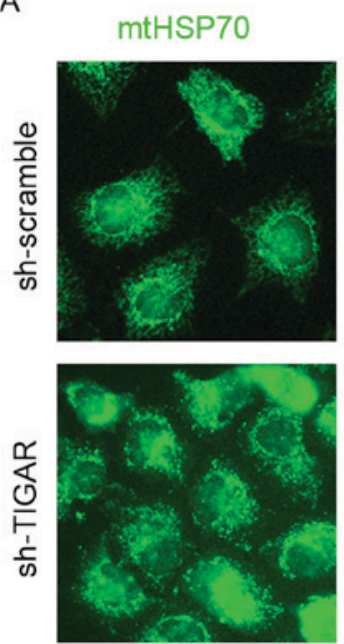

TIGAR
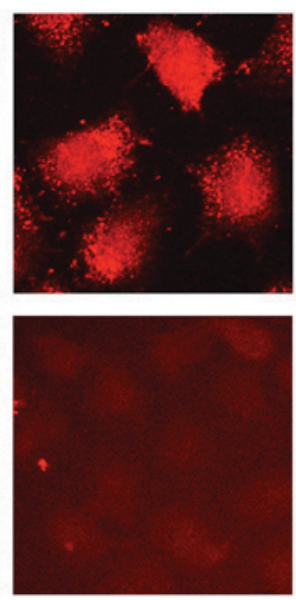

Merge
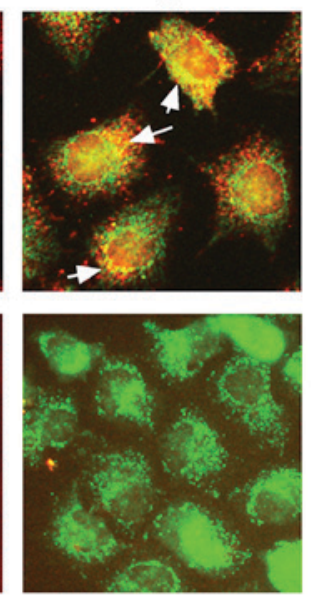

B

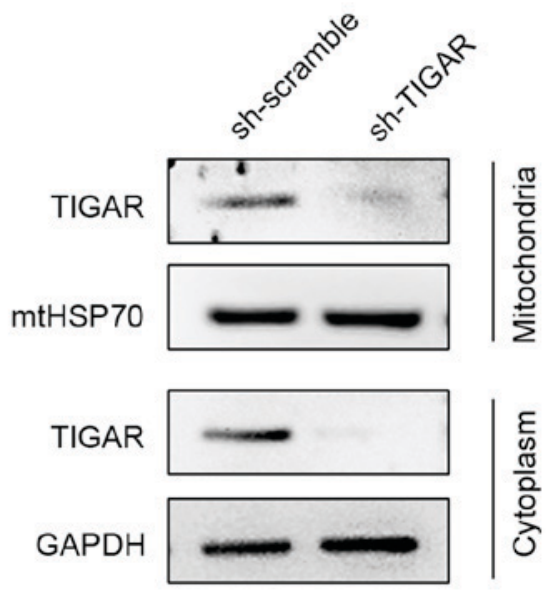

Figure 1. TIGAR localizes to mitochondria in 5-8F cells. (A) Immunofluorescence analysis of the mitochondria marker mtHSP70 (green) and TIGAR (red). TIGAR localizes to mitochondria as indicated by the white arrow in the upper panel. Lentivirus-mediated knockdown of TIGAR is shown in the lower panels. Original image magnification, $\mathrm{x} 400$. (B) Mitochondrial and cytoplasmic proteins were separated and subjected to western blot analysis to demonstrate localization of TIGAR in mitochondria. TIGAR, TP53-induced glycolysis and apoptosis regulator; sh, short hairpin; mtHSP70, mitochondrial heat shock protein 70 .

High-performance liquid chromatography analysis (HPLC). HPLC (Agilent Technologies, Inc., Santa Clara, CA, USA) was performed to determine the levels of ATP. The reverse phase consisted of a C18 column (4.6x150 mm; particle size, $3.5 \mathrm{~m})$. The mobile phase comprised buffer A $\left(15 \mathrm{mM} \mathrm{KH}_{2} \mathrm{PO}_{4}\right)$ and buffer B and methanol (90:10 ratio). A total of $20 \mu \mathrm{l}$ sample or standard was injected into the column. The flow rate was $0.5 \mathrm{ml} / \mathrm{min}$, and column temperature was $25^{\circ} \mathrm{C}$. The samples were analyzed by ultra violet detection at $254 \mathrm{~nm}$.

Transmission electron microscopy. The cells were pre-fixed with a mixed solution of $3 \%$ glutaraldehyde, then post-fixed in $1 \%$ osmium tetroxide, dehydrated in a series of acetone $(50,70,80$ and $90 \%$ acetone each for $15 \mathrm{~min}$ then $100 \%$ twice for $20 \mathrm{~min}$ ), infiltrated and embedded in EM bed812 (Electron Microscopy Sciences, Inc., Hatfield, PA, USA) at $37^{\circ} \mathrm{C}$ for $12 \mathrm{~h}, 45^{\circ} \mathrm{C}$ for $12 \mathrm{~h}$ and $60^{\circ} \mathrm{C}$ for $24 \mathrm{~h}$. The semi-thin sections $(5-\mu \mathrm{m}$ thick) were stained with methylene blue and ultra-thin sections (50-nm thick) were cut using a diamond knife, stained with uranyl acetate for $30 \mathrm{~min}$ followed by lead citrate for $10 \mathrm{~min}$ at room temperature. The sections were examined using a transmission electron microscope (Hitachi, Ltd., Tokyo, Japan; H-600IV).

Statistical analysis. All experiments were performed at least in triplicate. The results are expressed as the mean \pm standard deviation. Comparisons of flow cytometry results were performed using two-way analysis of variance and post-hoc Tukey's test. Comparisons of ATP content were performed using a paired Student's t-test. Statistical analysis was performed using SPSS 16.0 software (SPSS, Inc., Chicago, IL, USA) and GraphPad Prism 5.0 (GraphPad Software, Inc., La Jolla, CA, USA). P<0.05 was considered to indicate a statistically significant difference.

\section{Results}

TIGAR localizes to mitochondria in 5-8F cells. In the present study, the expression pattern of TIGAR in $5-8 \mathrm{~F}$ cells was detected using immunofluorescence staining, which demonstrated its partial localization on mitochondria marked by mtHSP70 (Fig. 1A). To confirm mitochondrial and cytoplasmic localization, mitochondrial and cytoplasmic proteins were separated and subjected to western blot analysis (Fig. 1B). To investigate the function of TIGAR on mitochondrial function, lentivirus was used to introduce shRNA (lenti-shRNA), which targets the TIGAR gene into 5-8F cells, and consequently marked reduction in the levels of TIGAR was observed in the mitochondria and cytoplasm, which strongly supported mitochondrial and cytoplasmic localization of TIGAR (Fig. 1).

Knockdown of TIGAR leads to loss of $\triangle \Psi$ min 5-8F cells. Decreased $\Delta \Psi \mathrm{m}$ can be detected by $\Delta \Psi \mathrm{m}$-dependent mitochondrial fluorescent probe, JC-1, and is a sensitive indicator of mitochondrial damage and dysfunction. In normal conditions, JC-1 aggregates to mitochondria and reflects red fluorescence, whereas it transfers to the cytoplasm as monomers and reflects green fluorescence when the $\Delta \Psi \mathrm{m}$ is decreased. Therefore, $\Delta \Psi \mathrm{m}$ can be reflected by the transition in fluorescence. Upon knockdown of TIGAR in $5-8 \mathrm{~F}$ cells, the ratio of red to green fluorescence decreased, indicating a reduction in $\Delta \Psi \mathrm{m}$. CsA, acalcineurin inhibitor that has the ability to maintain $\Delta \Psi \mathrm{m}$, was added to rescue the reduction in $\Delta \Psi \mathrm{m}$. The CCCP was used as a positive control to reduce $\Delta \Psi \mathrm{m}$ in the present study (Fig. 2A).

When $\Delta \Psi \mathrm{m}$ is reduced, the mtPTPs open, which leads to the leakage of mitochondrial content, including cytochrome c (14). In the present study, the levels of cytochrome c following $\Delta \Psi \mathrm{m}$ reduction in respective mitochondrial and cytoplasmic proteins were analyzed by western blot analysis. Knockdown of TIGAR led to increased levels of cytochrome $\mathrm{c}$ in the cytoplasmic fraction compared with control group, suggesting a mitochondrial leak (Fig. 2B).

Knockdown of TIGAR increases mitochondrial mass by preventing mitochondrial degradation. In addition to 
A
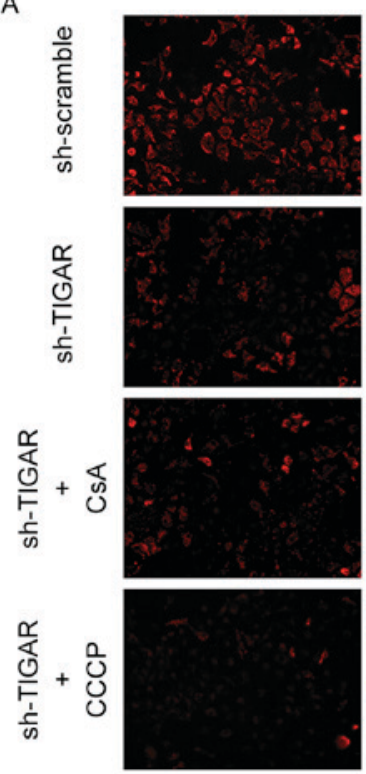
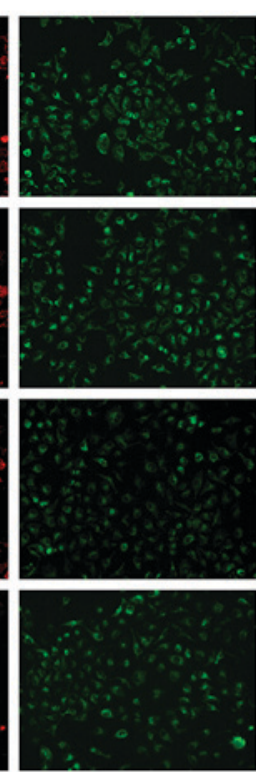
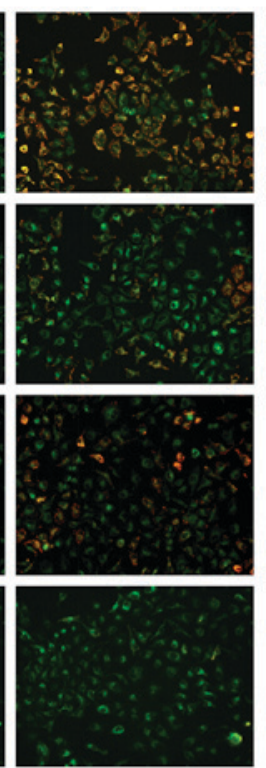

B

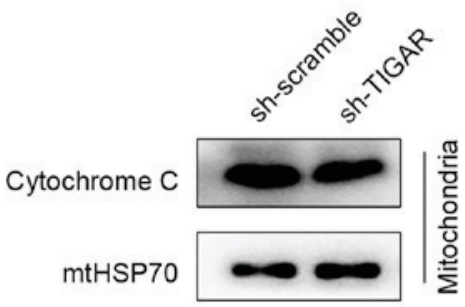

Cytochrome C

GAPDH
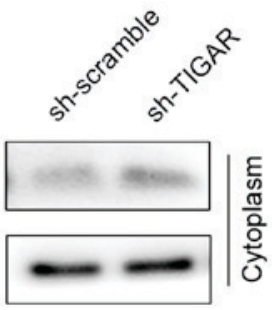

Figure 2. Knockdown of TIGAR leads to reduction in mitochondrial membrane potential and cytochrome c leakage in 5-8F cells. (A) The cells were incubated with CsA $(5 \mu \mathrm{M})$ and $\mathrm{CCCP}(5 \mu \mathrm{M})$ for $2 \mathrm{~h}$, and then mitochondrial membrane was determined by fluorescence microscopy using JC-1. The transition from red to green indicates reduction in mitochondrial membrane. Original image magnification, x200. (B) Mitochondrial and cytoplasmic proteins were separated to detect cytochrome $\mathrm{c}$ levels. TIGAR, TP53-induced glycolysis and apoptosis regulator; CsA, Cyclosporin A; CCCP, protonophore carbonyl cyanide m-chlorophenylhydrazone; sh, short hairpin.

the reduction in $\Delta \Psi \mathrm{m}$, it was further investigated in the present study whether mitochondrial mass was affected in TIGAR-knocked down cells. For these analyses, Mito-tracker Green, a dye that stains mitochondria independent of the $\Delta \Psi \mathrm{m}$, was used as aforementioned. Notably, it was indicated that the knockdown of TIGAR was able to increase the mitochondrial mass, regardless of the reduced $\Delta \Psi \mathrm{m}$ (Fig. 3A). To confirm this result, whole cell lysates were extracted from $5-8 \mathrm{~F}$ cells to determine the levels of the mitochondrial housekeeping protein, mtHSP70, which can reflect the mitochondrial mass in these cells. It was demonstrated that in TIGAR-knocked down cells, the level of mtHSP70 increased compared with the control cells (Fig. 3B), which was associated with the increase in mitochondrial mass observed (Fig. 3A).

To investigate whether degradation of damaged mitochondria was affected in TIGAR-knocked down cells, CCCP was added to $5-8 \mathrm{~F}$ cells to induce mitochondrial damage and degradation. Following a $2 \mathrm{~h}$ incubation with $\mathrm{CCCP}$, mitochondrial mass was significantly decreased in the control cells, whereas in TIGAR-knocked down cells mitochondrial mass was maintained (Fig. 3A and B). This suggested that TIGAR serves a crucial role in mitochondrial degradation.

Knockdown of TIGAR affects mitochondrial structure and physiological function. In the present study, reduction in $\Delta \Psi \mathrm{m}$ was able to prevent degradation of mitochondria in $5-8 \mathrm{~F}$ cells. In addition, to detect the integrity of mitochondria, the ultrastructure of mitochondria was analyzed by transmission electron microscopy. It was demonstrated that in TIGAR-knocked down cells, the mitochondria had an abnormal appearance and were observed to exhibit characteristics, including mitochondrial swelling, crista collapse and vacuolization (Fig. 4A). Furthermore, the ability to produce ATP was affected in these cells, indicating physiological dysfunction (Fig. 4B).
A
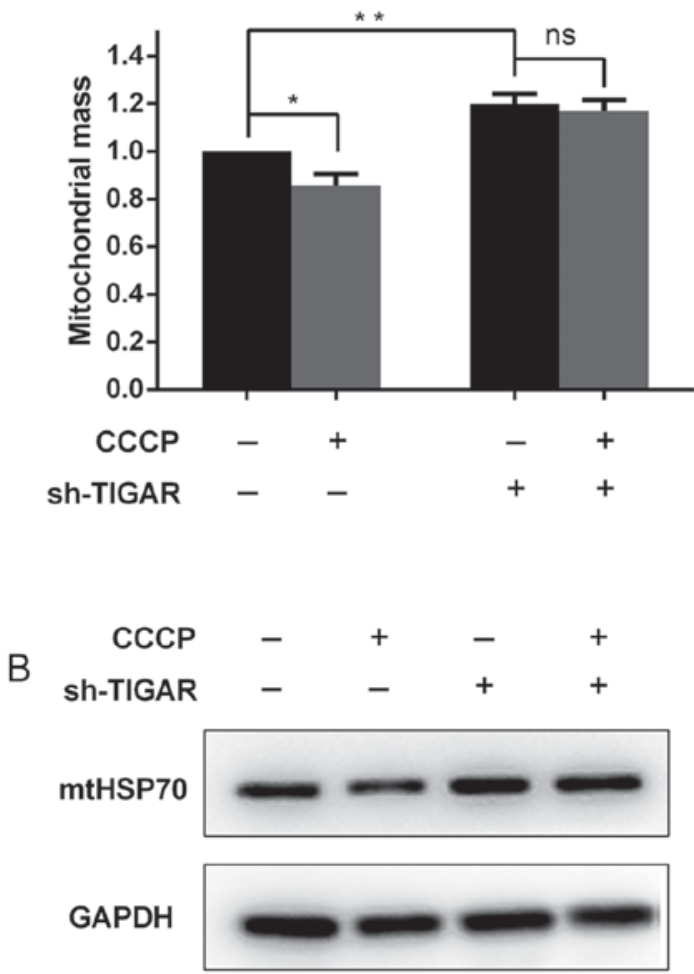

Figure 3. Knockdown of TIGAR increases mitochondrial mass by preventing degradation. (A) The cells were incubated with $5 \mu \mathrm{M} \mathrm{CCCP} \mathrm{for} 2 \mathrm{~h}$ and stained using Mitotracker Green $(50 \mathrm{nM})$, followed by detection using flow cytometry. The results are expressed as the mean \pm standard deviation from at least three independent experiments. Comparisons were performed using two-way analysis of variance and post-hoc Tukey's test. ${ }^{*} \mathrm{P}<0.05,{ }^{* *} \mathrm{P}<0.01$. (B) The levels of mtHSP70 and GAPDH protein were analyzed by western blotting. TIGAR, TP53-induced glycolysis and apoptosis regulator; CCCP, protonophore carbonyl cyanide $\mathrm{m}$-chlorophenylhydrazone; sh, short hairpin; ns, not significant; mtHSP70, mitochondrial heat shock protein 70 . 
A
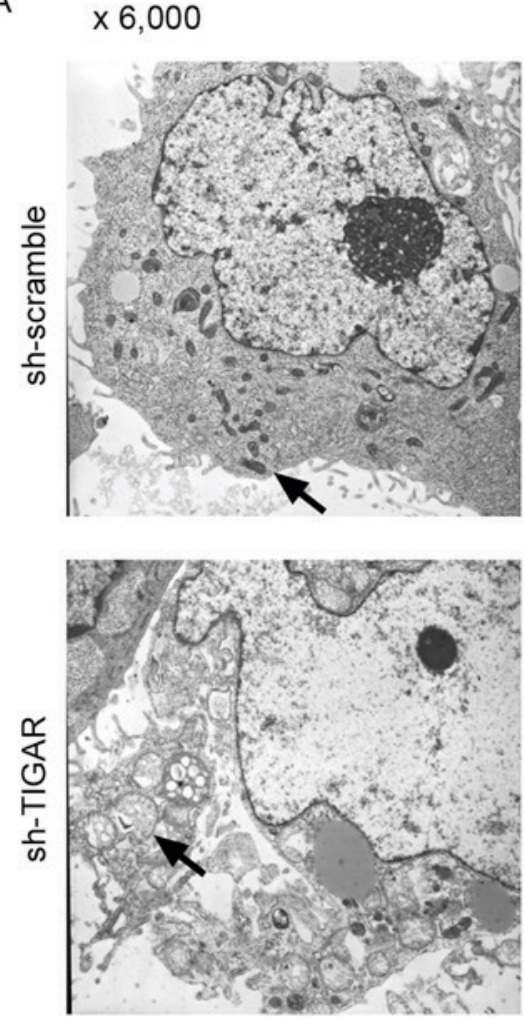

$x 15,000$
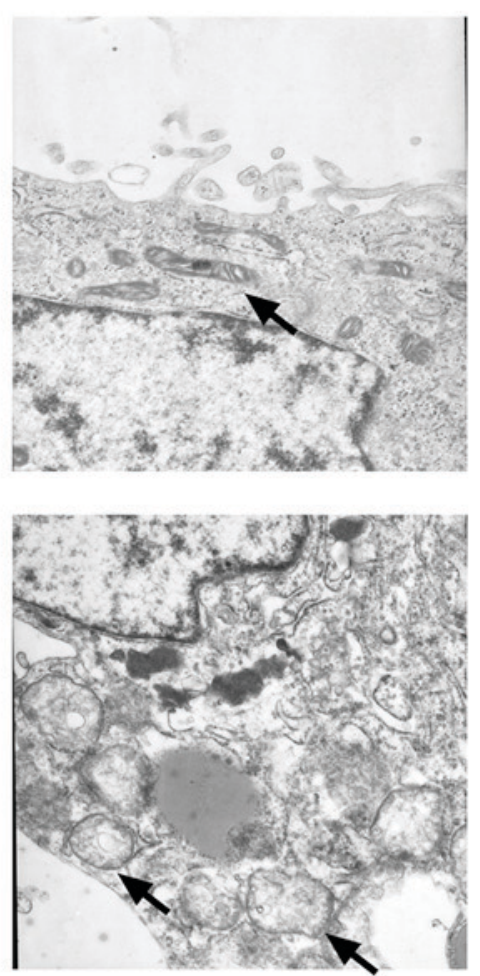

B

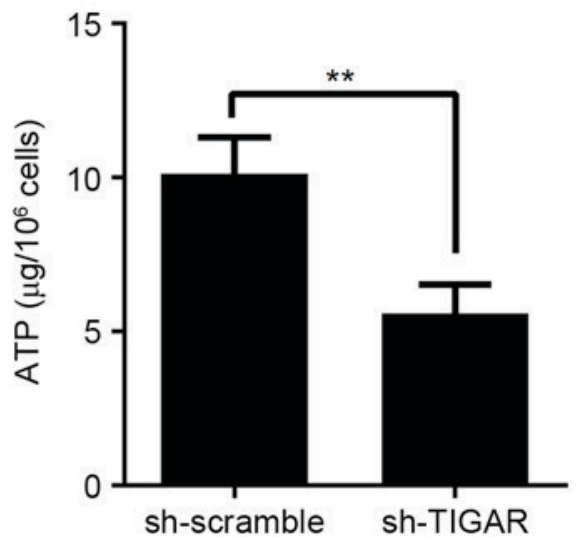

Figure 4. Knockdown of TIGAR affects mitochondrial structure and physiological function. (A) Electron micrographs reveal the ultrastructure of mitochondria in 5-8F cells. Mitochondrial swelling, crista collapse and vacuolization are indicated by the arrow in the lower panel. (B) The ATP content was evaluated by high-performance liquid chromatography. The results are expressed as the mean \pm standard deviation from at least three independent experiments. Comparisons were performed by a paired t-test. ${ }^{* *} \mathrm{P}<0.01$. TIGAR, TP53-induced glycolysis and apoptosis regulator; sh, short hairpin; ATP, adenosine triphosphate.

\section{Discussion}

TIGAR has the ability to limit fructose-2, 6-bisphosphatase levels, which results in a shift from glycolysis to PPP (11). $\mathrm{NADPH}$, as the product of the PPP pathway, serves an important role in ROS scavenging (11). As expected, a number of previous studies have reported that the knockdown of TIGAR is able to decrease NADPH production and reduce glutathione, thus contributing to ROS accumulation $(12,15,16)$. Simultaneously, dysregulated mitochondrial activity resulted in production of ROS as a by-product $(17,18)$. In the present study, it was detected that the loss of TIGAR leads to increased ROS levels (data not shown), which may cause irreversible damage to bio macromolecules and organelles (6).

Mitochondria are the source and target of ROS. Mitochondrial quality (structural and functional integrity) is essential forcellularfunction. Damaged mitochondria should be cleared in time, otherwise they may lead to metabolic diseases, including cancer (5). In the present study, notable reduction in $\Delta \Psi m$ and cytochrome c leakage in TIGAR-knocked down cells was observed, which suggested the initiation of mitophagy $(19,20)$. Of note, instead of clearance of mitochondria, an increase in mitochondrial mass was observed. For the degradation of mitochondria, a series of events is required to occur: i) Mitochondrial damage associated with membrane potential reduction; ii) an isolation membrane encircles damaged mitochondria to form double-membrane vesicles known as autophagosomes and iii) fusion with lysosome to digest its contents (21). TIGAR may serve an unknown role in the final two steps of the process. However, this is beyond the scope of the present study and requires further investigation.

In the present study, RNA interference was employed to investigate the potential effects of TIGAR on mitochondrial function in $5-8 \mathrm{~F}$ cells. In the present study, it was revealed that TIGAR is localized on mitochondria. In addition, the knockdown of TIGAR resulted in reduced $\Delta \Psi m$ and leakage of cytochrome $\mathrm{c}$ from the mitochondria to the cytoplasm. The mass of mitochondria was further determined, which was unexpectedly increased in TIGAR-knocked down cells. This increase in mitochondrial mass was associated with abnormal mitochondrial characteristics, including mitochondrial swelling, crista collapse and vacuolization. In addition, mitochondrial physiological dysfunction was demonstrated as indicated by the reduction in ATP production. In conclusion, TIGAR affects mitochondrial integrity and degradation in $5-8 \mathrm{~F}$ cells.

\section{Acknowledgements}

The present study was supported by the Scientific Research Foundation of the Education Department of Sichuan Province (grant no. 15ZA0163), the Affiliated Hospital of Southwest Medical University Foundation (grant no. 201519) and the Southwest Medical University Foundation (grant no. 20130388). 


\section{References}

1. Rich PR: The molecular machinery of Keilin's respiratory chain Biochem Soc Trans 31: 1095-1105, 2003.

2. Wallace DC, Fan W and Procaccio V: Mitochondrial energetics and therapeutics. Annu Rev Pathol 5: 297-348, 2010.

3. McBride HM, Neuspiel M and Wasiak S: Mitochondria: More than just a powerhouse. Curr Biol 16: R551-R560, 2006.

4. Mammucari C and Rizzuto R: Signaling pathways in mitochondrial dysfunction and aging. Mech Ageing Dev 131: 536-543, 2010.

5. Goldman SJ, Taylor R, Zhang Y and Jin S: Autophagy and the degradation of mitochondria. Mitochondrion 10: 309-315, 2010.

6. Wallace DC: A mitochondrial paradigm of metabolic and degenerative diseases, aging and cancer: A dawn for evolutionary medicine. Annu Rev Genet 39: 359-407, 2005.

7. Ashrafi G and Schwarz TL: The pathways of mitophagy for quality control and clearance of mitochondria. Cell Death Differ 20: 31-42, 2013.

8. Sun Y, Vashisht AA, Tchieu J, Wohlschlegel JA and Dreier L: Voltage-dependent anion channels (VDACs) recruit Parkin to defective mitochondria to promote mitochondrial autophagy. J Biol Chem 287: 40652-40660, 2012.

9. Kawajiri S, Saiki S, Sato S, Sato F, Hatano T, Eguchi H and Hattori N: PINK1 is recruited to mitochondria with parkin and associates with LC3 in mitophagy. FEBS Lett 584: 1073-1079, 2010.

10. Vives-Bauza C, Zhou C, Huang Y, Cui M, de Vries RL, Kim J, May J, Tocilescu MA, Liu W, Ko HS, et al: PINK1-dependent recruitment of Parkin to mitochondria in mitophagy. Proc Natl Acad Sci USA 107: 378-383, 2010

11. Bensaad K, Tsuruta A, Selak MA, Vidal MN, Nakano K, Bartrons R, Gottlieb E and Vousden KH: TIGAR, a p53-inducible regulator of glycolysis and apoptosis. Cell 126: 107-120, 2006

12. Bensaad K, Cheung EC and Vousden KH: Modulation of intracellular ROS levels by TIGAR controls autophagy. EMBO J 28: 3015-3026, 2009.
13. Mercurio F and Manning AM: NF-kappaB as a primary regulator of the stress response. Oncogene 18: 6163-6171, 1999.

14. Hough MA, Silkstone G, Worrall JA and Wilson MT: NO binding to the proapoptotic cytochrome c-cardiolipin complex. Vitam Horm 96: 193-209, 2014.

15. Yin L, Kosugi M and Kufe D: Inhibition of the MUC1-C oncoprotein induces multiple myeloma cell death by down-regulating TIGAR expression and depleting NADPH. Blood 119: 810-816, 2012.

16. Ye L, Zhao X, Lu J, Qian G, Zheng JC and Ge S: Knockdown of TIGAR by RNA interference induces apoptosis and autophagy in HepG2 hepatocellular carcinoma cells. Biochem Biophys Res Commun 437: 300-306, 2013.

17. Murphy MP: How mitochondria produce reactive oxygen species. Biochem J 417: 1-13, 2009.

18. Eng C, Kiuru M, Fernandez MJ and Aaltonen LA: A role for mitochondrial enzymes in inherited neoplasia and beyond. Nat Rev Cancer 3: 193-202, 2003.

19. Twig G, Elorza A, Molina AJ, Mohamed H, Wikstrom JD, Walzer G, Stiles L, Haigh SE, Katz S, Las G, et al: Fission and selective fusion govern mitochondrial segregation and elimination by autophagy. EMBO J 27: 433-446, 2008.

20. Lemasters JJ: Selective mitochondrial autophagy, or mitophagy, as a targeted defense against oxidative stress, mitochondrial dysfunction and aging. Rejuvenation Res 8: 3-5, 2005.

21. Levine B and Kroemer G: Autophagy in the pathogenesis of disease. Cell 132: 27-42, 2008.

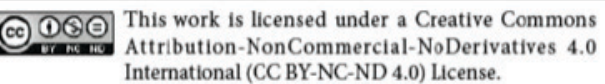
International (CC BY-NC-ND 4.0) License. 